\title{
Recommandations de bonnes pratiques professionnelles: entre positivisme et systémie, l'irruption de la complexité
}

\section{Roland Janvier}

\section{(2) OpenEdition}

Journals

Édition électronique

URL : http://journals.openedition.org/communicationorganisation/1525

DOI : 10.4000/communicationorganisation. 1525

ISSN : 1775-3546

Éditeur

Presses universitaires de Bordeaux

\section{Édition imprimée}

Date de publication : 1 décembre 2010

Pagination : 193-206

ISBN : 978-2-86781-743-4

ISSN : $1168-5549$

Référence électronique

Roland Janvier, «Recommandations de bonnes pratiques professionnelles: entre positivisme et systémie, l'irruption de la complexité », Communication et organisation [En ligne], 38 | 2010, mis en ligne le 01 décembre 2013, consulté le 19 avril 2019. URL : http://journals.openedition.org/ communicationorganisation/1525; DOI : 10.4000/communicationorganisation. 1525 


\title{
Recommandations de bonnes pratiques professionnelles : entre positivisme et systémie, l'irruption de la complexité
}

\author{
Rolond JANVIER 01
}

\section{Introduction}

Le Code de l'Action Sociale et des Familles prévoit, en son article L 312-8 que les établissements et services sociaux et médico-sociaux "procèdent à des évaluations de leurs activités et de la qualité des prestations qu'ils délivrent, au regard notamment de procédures, de références et de recommandations de bonnes pratiques professionnelles validées ou, en cas de carence, élaborées, selon les catégories d'établissements ou de services, par l'Agence nationale de l'évaluation et de la qualité des établissements et services sociaux et médico-sociaux.»

Il a fallu attendre 2002 pour voir s'imposer un principe général d'évaluation aux établissements et services sociaux et médico-sociaux. C'est donc tardivement, par rapport à de nombreux autres secteurs d'activité, que le travail social est entré dans la logique de l'évaluation de ses actions. Cette situation trouve deux types d'explication: culturelle et structurelle.

D'une part la culture des travailleurs sociaux semble très éloignée de l'idée de rendre compte de ses activités en formalisant les traces de ce qui est fait tant en amont, du côté des procédures, qu'en aval, de celui des rendus comptes. L'origine de cet état d'esprit est sans doute à rechercher dans ses fondations caritatives judéo-chrétiennes. "Faire le bien de l'autre » ne réclame en soi aucune autre justification que la bonne volonté de l'intention et la noblesse du geste, l'« inébranlable certitude d'être dans le vrai »(Karsz, 2004, p. 73).

01. Docteur en Sciences de l'Information et de la Communication (Université Rennes 2, Prefics), directeur général de la Fondation Massé Trévidy dans le Finistère laction sociale, lutte contre les exclusions, protection de l'enfance, handicap enfance, insertion par l'activité économique, enseignement agricole). Auteur de "Comprendre la participation des usagers dans les organisations sociales et médico-sociale (co-auteur Yves Matho, Dunod, 2011), "Conduire l'amélioration de la qualité en action sociale " (Dunod, 2009), "Éthique de direction en institution sociale et médico-sociale " (ESF, à paraître 2011). Roland Janvier a été membre du Conseil National de l'Évaluation Sociale et Médico-Sociale, dès 2005, puis de l'Agence Nationale de l'Évaluation Sociale et Médico-Sociale jusqu'en 2008. Mel: roland.janvier@gmail.com. Site: www.rolandjanvier.org 
Il aura fallu plusieurs siècles pour s'extraire de cette impasse relationnelle, reconnaître que les bénéficiaires ont aussi des droits, admettre les résistances organisées des groupements d'usagers et composer avec les demandes des intéressés. C'est, essentiellement, l'évolution du droit positif qui a engagé ce mouvement de reconnaissance de l'usager comme sujet de la relation d'aide (Janvier, Matho, 2004). Le corollaire de cette évolution se situe naturellement dans la mise en place de conditions de lisibilité, de visibilité et d'intelligibilité du travail social. L'évaluation constitue un élément de ce nouveau dispositif de traçabilité de l'action (Savignat, 2009) et le pivot d'une nouvelle légitimité (Janvier, 2009).

D'autre part, l'évaluation se heurte, quant il s'agit de "travail sur autrui » (Dubet, 2002), à une difficulté structurelle. Dans son acception vulgarisée, largement influencée par les qualiticiens de la production de biens matériels de consommation, l'évaluation se limite à un contrôle de conformité en référence à des critères qualitatifs. Il suffit de prévoir, de mettre en œuvre, de constater l'écart entre la prévision et la réalisation et de corriger les effets indésirables ou négatifs (cf. le Plan, Do, Check, Act de la roue de Deming). Ce modèle méthodologique - plus inspiré par la démarche qualité que par une réelle évaluation - se révèle inadapté à la relation d'aide. Les efforts de normalisation internationale pour ajuster les standards de qualité à la relation de service (cf. l'ancienne norme ISO 9002 intégrée maintenant dans la norme 9001-2000) ne sont pas encore réellement parvenus à prendre en compte l'ensemble des paramètres relationnels qui sont propres aux actions essentiellement fondées sur l'établissement d'un rapport intersubjectif. Les cadres référentiels, les méthodologies, les outils doivent donc être au moins repensés et au mieux créés pour s'adapter au cadre d'action spécifique dont il s'agit.

Dans ce contexte, l'introduction de l'obligation d'évaluer la qualité des prestations a été ressentie par les uns comme une insupportable injonction qui porte atteinte à la nature indicible du « colloque singulier » de la relation d'aide. D'autres y ont vu l'opportunité de simplifier des pratiques perçues comme compliquées et peu compréhensibles. Quels que soient les ressentis, la commande politique représente bien une taylorisation du travail social par l'imposition de standards (les recommandations de bonnes pratiques professionnelles publiées par l'Agence Nationale de l'Évaluation et de la qualité des établissements Sociaux et Médico-sociaux - ANESM), une tendance à rendre univoque le décryptage de l'action (c'est le mouvement convergent que l'on peut constater dans l'évolution de l'offre sur le marché des référentiels d'évaluation interne pour les établissements et services sociaux et médico-sociaux) et un alignement des critères de jugement de la qualité des prestations (ce sera l'évolution prévisible des pratiques d'évaluation externe qui sera conduite dans les établissements et services par les évaluateurs habilités). 
L'objet de cet article est de dépasser ce premier niveau d'évidence pour montrer qu'entre l'intention initiale du législateur et les modes de réception par le champ professionnel, quelque chose de plus complexe qu'un jeu de cause à effet est en train de se produire.

L'hypothèse sous jacente à cette démonstration repose sur l'identification de deux approches épistémologiques qui se combinent dans un rapport dialectique: en ce qui concerne l'édiction des normes, une conception positiviste de liens de causalité (il suffirait de dire la norme pour qu'elle se déploie dans les pratiques); du côté des mises en œuvre, une réalité beaucoup plus marquée par des effets de systèmes (l'intégration des normes édictées transite par des traductions qui tendent à éparpiller la commande en de multiples effets non prévus).

En fait, cela ne fonctionne pas comme prévu parce que le travail sur autrui renvoie inévitablement à la pensée complexe qui introduit la notion de système ouvert. Cette réalité constitue une ouverture stratégique, une occurrence, invitant les professionnels à se saisir du potentiel de la situation (Julien, 1996).

\section{Recommander des bonnes pratiques: une tentative pour rationnaliser les comportements professionnels}

L'objectif du législateur était bien - et est toujours - de maîtriser les comportements professionnels en imposant des normes. C'est, à titre d'illustration, ce qui explique l'évolution de l'instance chargée de coordonner cette grande entreprise d'évaluation ${ }^{02}$. Au départ, le Conseil National de l'Évaluation Sociale et Médico-Sociale, institué par la loi 2002-2, était « composé de l'ensemble des représentants du champ médico-social. (Il y aura) les représentants du secteur public, ceux du secteur associatif, ainsi que ceux des salariés... ${ }^{03}$ ». Mais déjà, était imaginé un établissement public de l'État - c'est l'Agence Nationale de l'Évaluation et de la Qualité des Établissement et Services Sociaux et Médico-sociaux qui sera créée par la loi de financement de la sécurité sociale pour $2007^{04}$ - tel que l'évoquent, à l'époque, les débats parlementaires: «cette agence aurait non seulement pour vocation de définir les bonnes pratiques, mais aussi de vérifier (...) que les prestations sont bien de la qualité qui est demandée..$^{05}$ "L'intention normative était affichée, confirmée dans les deux chambres et autant par l'opposition que par la majorité: "L'article 15 aborde un thème essentiel, à nos yeux, celui de l'évaluation, thème qui n'était pas pris en considération dans la loi de 1975. Il nous renvoie à la maltraitance, aux mauvaises pratiques

02. L'évaluation des établissements et services sociaux et médico-sociaux concerne plus de 32000 entités. 03. P. Terasse, député de la majorité, débats à l'Assemblée Nationale, séance du 31 janvier 2001.

04. Loi n 2006-1640 du 21 décembre 2006 de financement de la sécurité sociale pour 2007.

05. R. Bachelot-Narquin, députée de l'opposition, débats à l'Assemblée Nationale, séance du 31 janvier 2001. 
professionnelles et au manque de qualification qui justifient une évaluation objective extérieure à l'établissement afin de garantir le respect des bonnes procédures professionnelles. ${ }^{06}$ " D'ailleurs, la mise en place de l'ANESM, en mars 2007, s'inscrit dans le cadre du plan de développement de la bientraitance et de renforcement de la lutte contre la maltraitance: "Par son appui et ses recommandations, l'Agence constitue un élément clé du dispositif de mise en place effective des démarches de bientraitance. Elle est également essentielle dans le dispositif de lutte contre la maltraitance, car elle permettra la mise en œuvre de l'évaluation obligatoire et régulière des établissements, avec une évaluation réalisée par les établissements eux-mêmes et une évaluation externe sous son contrôle. ${ }^{07}$ " La suite de l'histoire atteste de l'intention politique d'investir l'ANESM d'un rôle coercitif de normalisation des pratiques ${ }^{08}$. Intention à laquelle se soumettra l'agence, au moins partiellement. Par exemple, en octobre 2008, elle s'inscrit dans l'opération «Bientraitance des personnes âgées accueillies en établissement » à la demande de la Secrétaire d'État à la Solidarité, Madame Valérie Létard. Elle diffusera un questionnaire d'« autoévaluation de la bientraitance des personnes âgées accueillies en établissement» ainsi qu'un guide de remplissage. Cette orientation des travaux de l'agence accrédite la thèse d'une fonction normative de cette instance. L'analyse du questionnaire en est un indice: « Renseigné par plus de $76 \%$ des établissements, ce questionnaire constitue un outil de dialogue et de transparence. Les établissements pourront eux-mêmes se situer et identifier leurs marges de progression, grâce à la synthèse nationale diffusée par l'Agence. Les résultats obtenus permettent aux autorités de définir des axes de travail nationaux et régionaux et d'engager des mesures d'accompagnement des établissements. » Le principe d'un classement des établissements en fonction de leur conformité aux références est, de fait, acté. L'ANESM ira-t-elle jusqu'à le réaliser?

Le concept même de recommandations de bonnes pratiques professionnelles révèle une conception tayloriste de la commande publique. Implicitement, la formule indique «la » bonne manière de procéder. Les précautions rhétoriques prises dans les textes et les déclarations ne peuvent tromper l'observateur attentif. Il s'agit bien d'une injonction. Cette posture

06. C-L. Campion, sénatrice, parti socialiste, débats au Sénat, séance du 31 octobre 2001.

07. Plan de développement de la bientraitance et de renforcement de la lutte contre la maltraitance, Ministère délégué à la Sécurité sociale, aux Personnes âgées, aux Personnes handicapées et à la Famille, 14 mars 2007

08. Suite à des faits de maltraitance dans des établissements de personnes âgées, Nora Bera, secrétaire d'État chargée des aînés, déclarait: " Je veux rendre obligatoire, au besoin par la Loi, la publication et la diffusion d'une évaluation indépendante et sérieuse, lisible pour nos concitoyens, de chacun des établissements susceptibles d'accueillir les personnes âgées dépendantes. Au regard de cette évaluation, je veux que les risques structurels de maltraitance au sein de ces établissements soient tout particulièrement évalués et quottés, pour être portés à la connaissance de chacun (....). Cette mission sera confiée à l'Agence Nationale de l'Évaluation et de la qualité des établissements et services Sociaux et Médico-sociaux, dont le rôle devra être amplifié. » (Discours du 2 décembre 2009). 
des décideurs politiques n'est pas surprenante, elle peut même être comprise comme étant inhérente à leur rôle. C'est la puissance publique qui, en France, confie par délégation, les missions d'utilité publique et d'intérêt général de l'action sociale et médico-sociale. Il est donc naturel qu'elle en fixe les contours et le contenu. Il faut cependant noter que c'est la première fois que la loi, et les dispositifs auxquels elle donne naissance, vont aussi loin dans la définition des pratiques professionnelles, des conduites à tenir. C'est la première fois qu'un système d'action se donne les moyens de conformer aussi précisément les attitudes attendues. Celles-ci se déclinent autour de quelques thèmes forts: la bientraitance (déjà évoquée dans les exemples précédents ${ }^{09}$ ), la performance (une Agence Nationale d'Appui à la Performance a été créée ${ }^{10}$ ), la maîtrise budgétaire (avec la généralisation plus ou moins annoncée de budgets réduits à un État Prévisionnel des Ressources et des Dépenses ${ }^{11}$ ), les référentiels professionnels ${ }^{12}$.

Tout ce mouvement descendant des décideurs vers les acteurs peut être interprété comme une tentative de simplification d'une activité sociale dans le but de faire coïncider les pratiques aux cadres référentiels préconstruits par les projections politiques.

\section{Les modes de réception des recommandations : un effet systémique}

Mais cela ne peut être aussi simple! Pour avoir une vision plus juste des évolutions en jeu, il faut également analyser les modes de réception par les acteurs professionnels des recommandations de bonnes pratiques professionnelles. Pour cela, nous allons centrer l'analyse sur le rôle d'interface que joue l'ANESM entre la commande politique et le champ professionnel.

La traduction opérationnelle des normes ne fonctionne pas selon une logique linéaire (cause/effet) mais par des effets de système (du type action/ rétroaction) marqués par des modalités d'appropriation, un rapport d'usage (le cadre limité de cet article ne permettra pas d'intégrer à l'analyse la manière concrète dont se déploient les recommandations dans les équipes de terrain).

Dès la mise en œuvre de l'évaluation issue de la loi rénovant l'action sociale et médico-sociale, le Conseil National de l'Évaluation Sociale et médicosociale a tenté d'infléchir la commande politique qui lui était faite. Dans sa seconde note d'orientation, en janvier 2006, après avoir fait une analyse sémantique des textes, tentant d'interpréter l'intention du législateur, le conseil tranche en déclarant qu'il «a la charge de valider les bonnes pratiques

09. Une recommandation centrale - dite "recommandation cadre" - a été publiée par l'ANESM sur ce thème: La bientraitance: définition et repères pour la mise en œuvre, juillet, 2008.

10. L'ANAP a été instituée par la loi n² 2009-879 du 21 juillet 2009 portant sur la réforme de l'hôpital et relative aux patients, à la santé et aux territoires.

11. Créé par l'ordonnance no 2005-406 du 2 mai 2005, décret no 2005-1474 du 30 novembre 2005 pour les établissements de santé.

12. Depuis quelques années, tous les métiers de l'action sociale se sont structurés autour de référentiels qui formalisent les compétences requises à l'exercice d'un métier. 
mais, ce faisant, il donne le cap sans entrer dans le détail de la mise en ceuvre du processus de l'évaluation, sans valider chacun des outils utilisables au cours de l'évaluation.» Il prend ainsi ses distances avec l'injonction normative qui lui est assignée: "la dénomination "bonne pratique professionnelle" peut susciter des craintes de standardisation et d'uniformisation et évoquer un cadre normé et desséchant qui étouffe la créativité des intervenants. " Il recentre finalement son ambition à la mise en évidence "des valeurs communes qui donnent sens aux actes professionnels et éclairent sur les perspectives de l'action. » Cette dernière phrase ré arrime la doctrine naissante de l'évaluation dans ce secteur d'activité aux cultures du champ professionnel.

Pour avoir été personnellement témoin de cette première période ${ }^{13}$ je peux confirmer la préoccupation première des représentants du secteur professionnel qui siégeaient au CNESM: elle consistait à rendre compatibles entre elles la commande publique, les attentes des acteurs de terrain, le besoin de développer une culture de l'évaluation, le tout en ne provoquant pas trop de réactions défensives ou de résistance. Le pari était complexe, il ne fut pas totalement gagné. En effet, les membres du CNESM - tout comme ceux qui composent aujourd'hui le Comité d'Orientation Stratégique de l'ANESM - ne forment pas un ensemble homogène. Plusieurs lignes de partages structurent les postures stratégiques du groupe, à l'image des divisions qui marquent le champ de l'action sociale et médico-sociale:

- Les types de publics et les modalités d'accompagnement créent des disparités fortes dans l'approche de l'évaluation. Entre un Établissement d'Hébergement pour Personnes Âgées Dépendantes, fortement médicalisé, et une équipe de prévention spécialisée, marquée par une faible institutionnalisation, la manière de penser l'évaluation de la qualité des prestations ne peut être identique.

Les statuts des structures d'intervention marquent également une grande différenciation. Entre le secteur public, le secteur lucratif et les organisations de l'économie sociale et solidaire, le rôle dévolu à l'évaluation est sensiblement différent. Dans l'un il s'inscrit dans une culture du service public, dans l'autre de la satisfaction des clients et dans les dernières, en référence à un projet social et politique.

Les idéologies sous jacentes aux interventions marquent également des écarts dans les conceptions de l'évaluation. Selon qu'une équipe se réfère aux théories psychanalytiques ou aux méthodes comportementales pour accompagner des personnes atteintes de troubles autistiques, l'investissement dans l'évaluation n'aura pas la même signification.

Certaines fédérations ont réalisé et vendent leur propre référentiel d'évaluation, d'autres ont créé des outils collaboratifs ouverts et évolutifs, d'autres enfin font preuve d'une grande méfiance à l'égard de référentiels livrés

13. Dès son installation, j'ai siégé au CNESM en tant que personne qualifiée, puis au Comité d'orientation Stratégique de l'ANESM à sa création et jusqu'en décembre 2008. 
" clés en main ». Ces divergences de positionnement entre les fédérations qui structurent - et divisent - le champ de l'action sociale et médico-sociale connotent les débats quant il s'agit de participer à la construction de références communes.

Les cultures professionnelles constituent également des lignes de fracture entre les acteurs. Entre la vision plutôt sanitaire des infirmiers, celle plus marquée par les dispositifs d'intervention des assistants de service social, la matrice éducative des éducateurs spécialisés, le regard social des animateurs ou l'approche économique des conseillers en économie sociale et familiale, ce sont autant de regards différents qui génèrent des avis particuliers.

C'est dans ce contexte que sont construites les recommandations de bonnes pratiques professionnelles ${ }^{14}$. De ce fait, parce que les modes de réception de l'injonction évaluative ne peuvent être univoques, le message se difracte dans les prismes d'interprétation très divers, parfois contradictoires, qui configurent le champ.

Du fait même de cette diversité, les recommandations vont, dès le départ, se montrer très prudentes: "Ces recommandations ne constituent pas une liste exhaustive d'exigences mais un outil de dialogue, de responsabilité, destiné a une mise en ceuvre adaptée selon les besoins et le contexte. ${ }^{15}$ " Il s'agit de ne braquer personne.

La légitimité des recommandations repose sur leur capacité à refléter les conceptions portées par les acteurs de terrain, et non pas à donner des leçons à des professionnels a priori très réticents. "Réalisée avec l'appui de compétences diversifiées, sur la base de travaux déjà menés dans les établissements et services, cette recommandation a pour objet de décrire la démarche [d'évaluation interne]. ${ }^{16}$ "

La dernière recommandation publiée, en octobre 2010, traite du questionnement éthique. Elle se fixe pour objectif «d'éprouver les démarches déjà en place, mais aussi d'expliquer, de partager et de confronter les valeurs portées par une structure au regard de celles auxquelles chaque professionnel adhère individuellement. ${ }^{17}$ » Le propos introductif situe clairement la recommandation au cœur d'un processus de débat selon une démarche bottom-up, non dans une logique top-down. L'exposé des objectifs de la recommandation éclaire encore mieux l'état d'esprit. Ils sont au nombre de trois:

- "Proposer une approche conforme aux spécificités qui caractérisent l'éthique, la différencie des normes juridiques, morales et déontologiques et permet d'en préciser le champ;

14. 17 recommandations sont publiées par l'ANESM entre avril 2008 et octobre 2010 : http://www.anesm. sante.gouv.fr/spip9242.html?page=article\&id_article $=375$

15. Recommandation "Expression et participation des usagers des établissements relevant du secteur de l'inclusion sociale», avril 2008, Introduction.

16. Recommandation "La conduite de l'évaluation interne dans les établissements et services sociaux et médico-sociaux visés à l'article L.312-1 du Code de l'Action Sociale et des Familles », juillet 2009, Introduction. 17. Recommandation « Le questionnement éthique dans les établissements et services sociaux et médicosociaux », octobre 2010, Introduction 
- Positionner la réflexion dans la pratique professionnelle en termes de bénéfices pour les personnes accompagnées et les professionnels;

- Proposer des repères méthodologiques pour mettre en place une réflexion éthique dans l'établissement. "

Quand l'ANESM parle de repères et d'approche, elle " propose », quand elle « positionne », il s'agit de la réflexion dans la pratique professionnelle et non de positionner ce qu'il convient de faire. Le propos est, pour le moins, attentif à ne pas se situer dans l'injonction. Il est symptomatique de la prudence avec laquelle le champ professionnel a accueilli ces recommandations, objets iconoclastes. D'ailleurs, le discours dominant des permanents de l'agence est bien de dire que les recommandations constituent des « repères pour l'évaluation », non des consignes à appliquer. De fait, la lecture attentive de l'ensemble des recommandations fait plutôt penser à un travail exhaustif de recensement de l'état des savoirs sur chaque question. Comment sont élaborées les recommandations? Une revue de littérature conduit à l'élaboration d'une liste d'items qui est ensuite travaillée par un groupe puis soumise soit à un protocole de consensus formalisé (groupe de cotation et groupe de lecteurs), soit à un consensus simple. Les deux démarches requièrent des enquêtes de terrain (aller voir au plus près des pratiques les savoirs faire déjà à l'œuvre) et la confrontation à des personnes ressources (chercheurs, auteurs de référence, acteurs reconnus).

Il existe donc un écart entre le niveau politique de l'intention, plutôt coercitive et normalisatrice et le niveau de la mise en œuvre, par l'agence elle-même, plutôt consensuelle et coopérative. Dans les faits, le rapport d'usage introduit par les modes d'appropriation des outils de l'évaluation fait émerger des dimensions imprévues par les concepteurs de la démarche. Les conduites des acteurs, tout en respectant la commande législative, contribuent correctement à stabiliser des normes d'usage, mais celles-ci sont plus ou moins éloignées de l'intention initiale des décideurs politiques.

Il est vraisemblable que la première instance de pilotage, le CNESM, a payé de sa vie une attitude jugée trop frileuse par les politiques. L'ANESM est née dans un contexte de vives tensions entre les représentants du secteur et le ministre Philippe BAS pour empêcher une remise au pas trop sévère. C'est l'UNIOPSS ${ }^{18}$ qui anime la résistance en adressant au nom d'un collectif une note au cabinet du ministre insistant sur la nécessaire prise en compte des particularités du champ et revendiquant que la future agence travaille non dans une démarche normative mais dans la suite des travaux engagés par le CNESM, réclamant une représentation de toutes les parties prenantes (nombre de fédérations et d'organisations représentatives d'usagers étaient évincées du projet de convention constitutive du Groupement d'Intérêt Public) et exigeant que prévale le même esprit de concertation qui avait été

18. Union Nationale Interfédérale et CŁuvres Privées Sanitaires et Sociales. 
la règle jusque là. C'est finalement une constitution de compromis qui sera validée. C'est le rôle central joué par le Comité d'Orientation Stratégique de l'ANESM, qui réussit à reprendre une place entre la direction de l'agence et le conseil scientifique qui permettra de résister à une simple mise en œuvre de l'injonction formelle du pouvoir politique.

Cette stratégie peut effectivement, au premier abord, être interprétée comme une résistance. Il s'agit en fait d'un phénomène plus complexe, plus systémique. Nous sommes en présence d'une traduction. La commande politique est, en quelque sorte, digérée par les grilles d'interprétation des acteurs. Les acteurs sont ici les représentants du secteur qui siègent au Comité d'Orientation Stratégique, les membres du conseil scientifique (dont beaucoup furent ou sont encore des professionnels de terrain), les professionnels appelés à travailler dans les groupes (pilotage, cotation, lecture, enquêtés), mais aussi les chargés de mission de l'agence dont les plus influents ont une expérience de terrain.

Cette opération de traduction est à la fois le préalable à une réception correcte - c'est-à-dire sans trop de «bruit»-par les cultures locales des acteurs et la condition d'une poursuite de l'activité sans créer de rupture avec les repères techniques qui permettent l'action.

\section{L'affirmation d'un monde ouvert dans un contexte de complexité}

En effet, et c'est sans doute là l'essentiel du phénomène, la tentation d'une rationalisation radicale des process, d'une taylorisation des références, serait un acte mortifère pour le travail social. Cette forme particulière de travail sur autrui ne pourrait vraisemblablement pas survivre dans un contexte totalement contraint par des normes fermées qui feraient clôture à l'imprévisible de toute relation humaine. C'est donc à la fois parce qu'il s'agit d'une condition indispensable à la survie d'un système complexe de pratiques et parce qu'il est impossible de réduire la relation d'aide à une codification binaire que l'injonction normative n'a pas pu fonctionner totalement et, nous en faisons le pari optimiste, qu'elle ne pourra jamais se réaliser complètement.

En fait, l'éclatement du principe de causalité descendante en une diffusion incontrôlable d'impacts imprévisibles sur les pratiques et les postures tient essentiellement à la réalité complexe dans laquelle s’inscrit le travail sur autrui.

$\mathrm{Du}$ conflit épistémologique entre la vision positiviste qui tente de rationnaliser les comportements professionnels et les modalités plus systémiques de l'appropriation des recommandations de bonnes pratiques par les acteurs de terrain, naît un éclairage fécond qui réhabilite la pensée complexe. La réflexion d'Edgar Morin va nous aider à tracer les contours de cette intuition qui ouvre des perspectives stratégiques.

C'est le propre de la pensée simplificatrice que de tenter d'abolir la diversité en unifiant par l'abstraction l'hétérogénéité des phénomènes. C'est ainsi que de nombreux référentiels d'évaluation se réduisent à n'être que 
des instruments de comptage. Mais on ne peut comptabiliser que ce qui est quantifiable et l'évaluation se borne alors à mesurer des écarts. Dans ce cas, les recommandations de bonnes pratiques professionnelles seront interprétées comme des consignes à appliquer, en totale indépendance des contextes où elles se vivent. Cette méthode, qui se veut unificatrice par simplification, ne fait que juxtaposer des éléments divers pour les dénombrer sans créer de lien signifiant entre eux. "Ainsi, on arriveà l'intelligence aveugle. L'intelligence aveugle détruit les ensembles et les totalités, elle isole tous ses objets de leur environnement. " (Morin, 2005, p. 19). La pensée simple procède par disjonction: elle sépare les éléments pour les réduire un par un à leur plus petite unité compréhensible. Mais elle procède également par réduction, c'est-à-dire qu'elle unifie ce qui est divers créant des ensembles incohérents dont la seule utilité est de donner l'impression d'un ensemble compréhensible. Par exemple, évaluer la qualité de l'accompagnement éducatif dans un Institut Médico Éducatif ne peut se faire en isolant l'établissement de son environnement. Évaluer ce qui se passe entre les murs sans prendre en compte les interactions avec les problématiques familiales, les écoles du territoire, le cadre de vie revient à n'observer qu'une partie d'une réalité bien plus complexe et à se priver de la comprendre. À l'inverse, évaluer les dimensions éducatives, thérapeutiques et pédagogiques de l'action en ne les différenciant pas revient à amalgamer des plans différents d'interprétations sans mettre en lumière la logique interne propre à chaque discipline.

La pensée complexe, quant à elle, va prendre en compte les constituants hétérogènes, voire incohérents, d'un système, «elle pose le paradoxe de l'un et du multiple » (ibid.p. 21). La pensée complexe permet d'appréhender les entrelacs d'évènements, d'actions, d'interactions, de rétroactions, de déterminations et d'indéterminations, d'aléas et de finalités prévues « qui constituent notre monde phénoménal»(ibid.). Selon ces perspectives, les références deviennent des repères, les protocoles des processus, les consignes des indications, bref, la pensée complexe introduit de la fluidité là où la vision positiviste rigidifie les choses. La lecture de référentiels d'évaluation selon ce critère d'analyse met en lumière ceux qui classent (bon/mauvais, bien/mal, permis/défendu...) et ceux qui ouvrent un espace de travail, de réflexion et de débat.

Fondamentalement, la relation d'aide, fondement de l'action sociale et médico-sociale, gagne à être pensée - théorisée - en référence à la pensée complexe parce que celle-ci s'appuie sur la notion de système ouvert. Ce concept, issu de la thermodynamique, nous parle de déséquilibre, de mouvement et de mouvance, d'échanges énergétiques. Il nous montre que l'intelligibilité d'un système réside dans la compréhension de ses relations à son environnement, de ce qui s'échange à sa périphérie et non pas uniquement de ce qui se passe en son sein. Penser l'institution sociale comme un système clos appelle une conception statique de son métabolisme. C'est le principe de conformité qui est alors convoqué. Puisque ce qui compte c'est ce qu'il est - ou 
ce qu'il a - et non ce qu'il échange, ce sont les attributs qu'il faut évaluer (on vérifie alors que l'établissement dispose bien d'un livret d'accueil, que le conseil de la vie sociale existe, que les réunions de synthèse ont lieu régulièrement, etc. sans s'intéresser à ce que ces objets ont comme effets dans le système). À l'inverse, penser les institutions sociales comme des systèmes ouverts revient à les considérer "comme des systèmes organisant leur clôture (c'est-à-dire leur autonomie) dans et par leur ouverture. " (Ibid. p. 31). Ils ne sont pas alors conçus comme des systèmes en équilibre dans lesquels les prescriptions normatives vont garantir la conformité mais comme des systèmes en déséquilibre qui ont besoin de s'alimenter de points de repères pour évaluer la qualité de leur mouvement.

L'évaluation, selon cette voie, n'est plus une injonction uniformisant les pratiques sur la base de standards exogènes, elle est un germe structural (Simondon, 2005) qui met en jeu les échanges énergétiques dans et autour de l'organisation, les redynamise au bénéfice d'une renégociation du rapport entre normes et formes (Janvier, 2007).

L'écart paradigmatique entre la conception d'un système clos et celle d'un système ouvert est un abîme sans fond. C'est ce vide sidéral qui sépare l'injonction normative d'une évaluation conçue comme œuvre de simplification de l'appropriation systémique des acteurs inscrite dans la complexité d'un système ouvert. Au centre de ce gouffre se trouve l'Agence Nationale de l'Évaluation et de la Qualité des Etablissements et Services Sociaux et Médico-sociaux. Son avenir paraît compromis à certains, prise ainsi au piège mortel d'une radicale contradiction. Or, son existence comme interface, espace de traduction, est essentielle. L'ANESM est un intermédiaire au sens des travaux de Michel Callon et Bruno Latour sur la sociologie de la traduction. Un intermédiaire, c'est: « toute entité (pouvant associer les différents éléments que nous avons eu l'occasion d'énumérer...), qui définit et construit un monde peuplé d'autres entités, les dote d'une histoire, d'une identité et qualifie les relations qui les unissent. »(Callon, 1991, p. 205).

L'ANESM est un acteur de traduction, c'est-à-dire "un intermédiaire auquel la mise en circulation d'autres intermédiaires est imputée.» (Ibid., p. 209). Sans elle, cette circulation complexe entre les parties prenantes ne serait plus possible. Linjonction normative deviendrait une dictature, plus aucun jeu ne serait possible entre la commande et sa mise en œuvre. Sans l'ANESM, le divorce serait définitivement consommé entre les décideurs politiques et le monde professionnel du travail social. Les conséquences de cette rupture seraient considérables.

Par sa présence, l'ANESM ne mène pas une action de résistance - qui pourrait imaginer qu'un Groupement d'Intérêt Public de l'État s'oppose à la commande publique légitime? -, elle fait œuvre d'apprentissage. L'apprentissage, selon Michel Callon, est "l'ensemble des mécanismes par lesquels, par progressive adaptation mutuelle et redéfnitions, les différents éléments 
pris dans une traduction deviennent exclusivement dépendants les uns des autres. " Cette dépendance réintroduit de la transduction (Simondon, 2005) là où la défaillance de l'ANESM ne laisserait que de la contrainte et de l'assignation selon un régime exclusivement prescriptif.

Cette clef théorique, de l'opposition paradigmatique, permet à l'observateur de comprendre la manière particulière dont l'évaluation est en train de se diffuser dans l'action sociale et médico-sociale.

\section{BIBLIOGRAPHIE}

CALLON Michel, Réseaux technico-économiques et irréversibilité, In R. Royer, B. Chavance, O. Godard (sous la dir.), "Les figures de l'irréversibilité en économie ", Éditions de l'École des Hautes Études en Sciences Sociales, 1991.

DUBET François, Le déclin des institutions. Paris, Le Seuil, 2002.

JANVIER Roland \& MATHO Yves, Mettre en ceuvre le droit des usagers dans les établissements d'action sociale. Paris, Dunod, 1999, 3 é édition 2004.

JANVIER Roland, Conduire l'amélioration de la qualité en action sociale, Communiquer, manager, organiser, agir, Paris, Dunod, 2009.

JANVIER Roland, L'évaluation des établissements et services sociaux et médico-sociaux: entre imaginaires d'usages et usagers, Communication au colloque MEOTIC, à l'Institut de la Communication et des Médias (Université Stendhal Grenoble), 7 et 8 mars 2007.

JULIEN François, Traité de l'efficacité. Grasset (livre de poche), Paris, 1996.

KARSZ Saül, Pourquoi le travail social? Paris, Dunod, 2004.

MORIN Edgar, Introduction à la pensée complexe, Paris, Le Seuil, 2005.

SAVIGNAT Pierre, Evaluer les établissements et les services sociaux et médico-sociaux, Paris, Dunod, 2009.

SIMONDON Gilbert. L'individuation à la lumière des notions de forme et d'information. Grenoble, éditions Jérôme Millon - collection Krisis, 2005.

\section{RÉSUMÉ :}

La généralisation de l'évaluation dans le champ de l'action sociale et médico-sociale répond à une demande de contrôle et de normalisation de l'État. Cette intention, fortement marquée par une conception positiviste, se concrétise dans des recommandations de bonnes pratiques professionnelles. La manière dont ces dernières s'écrivent et se mettent en œuvre dans les pratiques professionnelles révèle une logique de diffusion plus systémique que la simple relation de cause à effet. Ce sont les concepts de la pensée complexe qui permettent de discerner ce qui est à l'œuvre dans ce mouvement de diffusion. L'Agence Nationale de l'Évaluation et de la qualité Sociale et Médico-sociale joue le rôle d'intermédiaire réalisant un processus d'apprentissage qui relie la commande politique aux mises en œuvre de l'évaluation dans les établissements et services.

Mots clefs : évaluation, normalisation, standardisation, système, complexité. 


\begin{abstract}
Large development of evaluation in social-intervention field is an answer to the state requisite for control and standardization. This demand, which is strongly influenced by a positivist point of view, finds its practical application in recommendations for fair professional manners. The way within those advises are written and set up reveals a more systemics logic of diffusion, than that which would have prevailed if there has simply been a causality relation. And the striking concepts to enlighten the point of this diffusion movement are that of the "complexity thought". "L'Agence Nationale de l'Évaluation et de la qualité Sociale et Médicosociale" plays the part of an intermediary to launch a learning process between the political decisioning and the achievement of evaluation in the units and departments.
\end{abstract}

Keywords : evaluation, normalization (Standardization), system, complexity 